\title{
Understanding the ever-changing incidence of thyroid cancer
}

Cari M. Kitahara ${ }^{1 凶}$ and Julie A. Sosa ${ }^{2}$

This Comment article provides a behind-the-scenes perspective and update of our 2016 Review, which discussed possible factors contributing to thyroid cancer incidence trends worldwide. We also highlight promising research directions that are improving the understanding of thyroid cancer aetiology.

In 2016, we co-authored a Review for Nature Reviews Endocrinology, entitled 'The changing incidence of thyroid cancer ${ }^{1}$. In this Review (cited 332 times according to Google Scholar, as of 18 August 2020), we discussed the rising incidence of thyroid cancer in the USA and much of the rest of the world and the probable reasons behind the changes. The timing of our Review coincided with major shifts in thinking about the epidemiology, aetiology and clinical management of thyroid cancer, and we covered many of the key points raised at the 2015 Endocrine Society conference symposium on this topic (C.M.K. was a speaker and J.A.S. was the moderator). Bringing together our different areas of expertise (epidemiology, clinical thyroidology and endocrine surgery), we sought to provide a balanced, comprehensive discussion on the topic from both a public health and clinical perspective. In addition to discussing the important role of overdiagnosis in the rising rates of thyroid cancer worldwide, we also highlighted factors, such as obesity, that might have contributed to a true increase in the occurrence of this disease, particularly in the USA.

By 2016, the contribution of overdiagnosis to thyroid cancer incidence trends was well recognized. In the USA, the incidence of thyroid cancer, particularly papillary thyroid cancer (PTC), increased rapidly for several decades, beginning in the early 1980s, with an increasing proportion of smaller and early-stage versus larger and more advanced PTCs ${ }^{1}$. These trends were largely attributed to the more widespread use of diagnostic imaging and fine-needle aspiration biopsies of thyroid nodules since the 1980s and 1990s. Despite this shift towards diagnosis of tumours at reduced risk of disease recurrence or disease-specific mortality, there was also evidence of an increasing proportion of patients being treated aggressively with thyroidectomy along with radioactive iodine (RAI) ablation or RAI therapy ${ }^{2,3}$. At the same time, the substantial adverse psychological, financial and health consequences of overdiagnosis and overtreatment of thyroid cancer were becoming increasingly apparent.

Recognizing the urgent need to reverse these trends, between 2009 and 2017, the American College of
Radiology, the American Thyroid Association (ATA) and the US Preventive Services Task Force issued strong recommendations against biopsy of very small thyroid nodules and those lacking suspicious features and screening of thyroid cancer in the asymptomatic population ${ }^{4,5}$. For the first time, the 2015 ATA guidelines discussed use of molecular testing for nodules with indeterminate cytology to circumvent diagnostic surgery. For tumours lacking suspicious or aggressive features, alternative 'less is more' management strategies were called out, including active surveillance for papillary thyroid microcarcinomas, thyroid lobectomy as an alternative to total thyroidectomy for low-risk differentiated thyroid cancer and use of reduced administered activities (doses) of $\mathrm{RAI}^{5}$. In 2017, the ATA recommended a terminology change for noninvasive encapsulated follicular variant of PTC, from a malignant to an in situ neoplasm, owing to its excellent prognosis ${ }^{6}$. The extent to which changing clinical guidelines have or will influence trends in thyroid cancer incidence and mortality in the USA and elsewhere might not be fully apparent for several more years; however, the statistically significant decline in thyroid cancer incidence in the USA from 2015 to 2017 suggests that these efforts have already had a major effect ${ }^{7}$.

Our Review also summarized evidence suggesting that enhanced detection, or overdiagnosis, could explain only about half of the rise in PTC incidence in countries such as the USA and Australia, due to the trends observed for larger and more advanced PTCs, as well as thyroid cancer mortality ${ }^{1}$. As a follow-up to this discussion, in 2017, our research team published a comprehensive analysis of US cancer registry data, which confirmed that the increasing incidence of PTCs between the mid-1970s and mid-2010s was not exclusive to tumours that were small and localized at diagnosis and that the increase in incidence was accompanied by a more subtle but statistically significant increase in thyroid cancer mortality ${ }^{8}$. We also found that the increase in thyroid cancer mortality was exclusive to patients diagnosed with PTC and was most apparent for those diagnosed with advanced PTC. Thus, in addition to overdiagnosis, we concluded that there appeared to be a true, concurrent increase in the 
Total papillary thyroid cancer

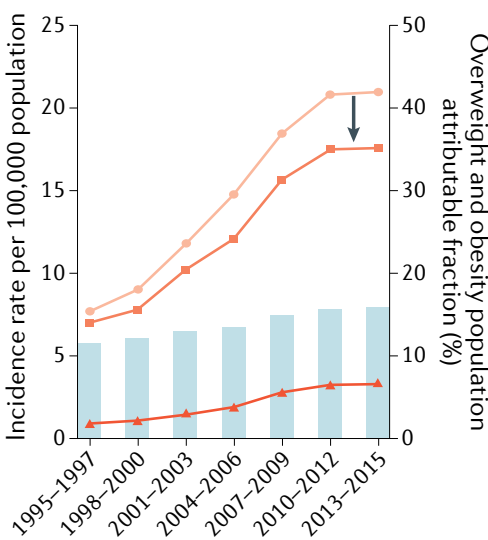

Total

Population attributable fraction
Papillary thyroid cancers $>4 \mathrm{~cm}$

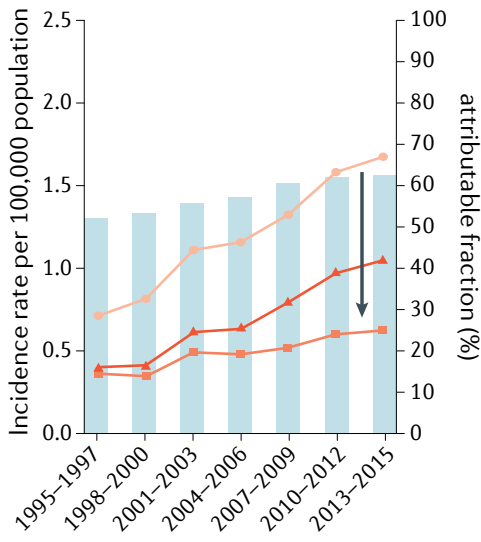

- Unrelated to obesity or overweight $\neg$ Related to obesity or overweight

Fig. 1 Age-standardized incidence rates of papillary thyroid cancer (overall and large $(>4 \mathrm{~cm})$ among people aged $\geq 60$ years in the US Surveillance, Epidemiology, and End Results - 13 cancer registry program, 1995-2015). The rates of papillary thyroid cancer are shown as overall rates and have been stratified as attributable to overweight or obesity or as being unrelated to overweight or obesity. Annual population attributable fractions are based on relative risk estimates from the NIH-AARP Diet and Health Study and prevalence estimates of overweight and obesity among US adults aged $\geq 50$ years from the National Health Interview Survey (1985-2005). The arrows represent the hypothetical reduction in the incidence rates for papillary thyroid cancer in the absence of overweight and obesity in the 10 years before the date of diagnosis. Please note the 10 -fold difference in scale of the $y$ axes. Adapted with permission from REF. ${ }^{10}$, Oxford University Press.

occurrence of thyroid cancer. These results lent further support to the hypothesis that changes in the prevalence of modifiable risk factors might have at least partially contributed to observed trends in thyroid cancer in the USA, and possibly other countries as well.

We also discussed potential environmental and lifestyle-related factors that might have contributed to observed thyroid cancer incidence and mortality trends ${ }^{1}$. Since the publication of our Review, the evidence supporting an association between obesity and increased risk of thyroid cancer, particularly PTC and anaplastic thyroid cancer, has continued to grow. For example, studies have shown that obesity might be more strongly associated with the risk of PTC characterized by suspicious or aggressive tumour features than PTC lacking these features ${ }^{9}$. Combining information from cohort, survey and cancer registry data, our research team estimated that about $16 \%$ of all PTCs (63\% of large PTCs) diagnosed in 2013-2015 in the USA were attributable to overweight and obesity ${ }^{10}$. In the absence of overweight and obesity, PTC incidence trends would have been attenuated by about 14\% (58\% for large PTCs) (FIC. 1). These results suggest that public health measures aimed at minimizing excess weight might help to prevent the development of PTC, among numerous other health benefits.

Further progress towards a better understanding of thyroid cancer aetiology will require overcoming some of the inherent challenges of existing observational studies and original, outside-the-box thinking. Large, wellpowered and carefully designed case-control and cohort studies are needed to precisely quantify associations

between exposures and thyroid cancer risk; however, few such studies currently offer the ability to distinguish between true risk factors versus risk factors for overdiagnosis. One exception is the Queensland Thyroid Cancer Study ${ }^{9}$, in which clinical records, pathology data and tumour tissues were collected to obtain information about the method of initial thyroid cancer detection and diagnosis and characteristics of the tumours. This level of diagnostic information enables separate evaluation of exposuredisease relationships for higher-risk versus lower-risk thyroid cancers. Novel aetiological factors might yet be discovered through exploration of rich, unique data resources and by investigating exposures occurring during potentially susceptible periods in the life course. Laboratory-based studies and observational studies with pre-diagnostic biomarkers of exposure are also valuable in understanding biological pathways underlying observed associations. Finally, comprehensive descriptive epidemiological studies continue to play an understated but pivotal role, not only in guiding future clinical recommendations but in generating hypotheses regarding factors that might be contributing to changing incidence and mortality trends. Such studies could also be used to quantify the effect of the novel coronavirus (COVID-19) pandemic on thyroid cancer diagnostic and treatment practices in 2020 and beyond.

In conclusion, we thank the editors of Nature Reviews Endocrinology for the opportunity to express our views on this topic, as well as the four peer reviewers who provided valuable feedback on our original Review. We hope that our previous and current commentaries inspire future descriptive, aetiological and clinical research on thyroid cancer and illustrate the value of multidisciplinary collaborations among researchers sharing the common goals of thyroid cancer prevention and improved patient outcomes.

1. Kitahara, C. M. \& Sosa, J. A. The changing incidence of thyroid cancer. Nat. Rev. Endocrinol. 12, 646-653 (2016).

2. Sosa, J. A. et al. Increases in thyroid nodule fine-needle aspirations, operations, and diagnoses of thyroid cancer in the United States. Surgery 154, 1420-1426 (2013).

3. Haymart, M. R. et al. Use of radioactive iodine for thyroid cancer. JAMA 306, 721-728 (2011).

4. Haugen, B. R. 2015 American Thyroid Association Management Guidelines for adult patients with thyroid nodules and differentiated thyroid cancer: what is new and what has changed? Cancer 123 . 372-381 (2017)

5. US Preventive Services Task Force. Screening for thyroid cancer: US Preventive Services Task Force Recommendation Statement. JAMA 317, 1882-1887 (2017).

6. Haugen, B. R. et al. American Thyroid Association guidelines on the management of thyroid nodules and differentiated thyroid cancer task force review and recommendation on the proposed renaming of encapsulated follicular variant papillary thyroid carcinoma without invasion to noninvasive follicular thyroid neoplasm with papillary-like nuclear features. Thyroid 27, 481-483 (2017)

7. Lee, M. et al. Letter to the Editor: reversal in thyroid cancer incidence trends in the United States, 2000-2017. Thyroid 30, 1226-1227 (2020).

8. Lim, H. et al. Trends in thyroid cancer incidence and mortality in the United States, 1974-2013. JAMA 317, 1338-1348 (2017).

9. Rahman, S. T. et al. Obesity is associated with $B R A F^{V 600 E}$-mutated thyroid cancer. Thyroid https://doi.org/10.1089/thy.2019.0654 (2020).

10. Kitahara, C. M. et al. Impact of overweight and obesity on US papillary thyroid cancer incidence trends (1995-2015). J. Natl Cancer Inst. 112, 810-817 (2020).

\section{Acknowledgements}

The authors acknowledge the support of the Intramural Research Program of the National Cancer Institute at the National Institutes of Health.

\section{Competing interests}

J.A.S. is a member of the Data Monitoring Committee of the Medullary Thyroid Cancer Consortium Registry supported by GlaxoSmithKline, Novo Nordisk, AstraZeneca and Eli Lilly. J.A.S. receives institutional research funding from Exelixis and Eli Lilly. C.M.K. declares no competing interests. 